\title{
Una ciencia política transdisciplinar en Colombia ${ }^{1}$
}

\section{A transdisciplinary political science in Colombia}

Recibido: 21 de abril de 2014 - Revisado: 01 de febrero de 2015 - Aceptado: 01 de abril de 2015

Julián Andrés Caicedo Ortiz ${ }^{2}$

Sergio Angel Baquero ${ }^{3}$

Julián Andrés Cuellar Argote ${ }^{4}$

\section{Resumen}

El artículo propone una interpretación epistemológica sobre el desarrollo disciplinar de la ciencia política en Colombia. A través de un ejercicio hermenéutico se fundamenta la necesidad de pensar y reconocer la ciencia política de manera (trans) disciplinar, para luego mostrar que en el proceso de conformación de las distintas plantas docentes han participado profesionales de diferentes ramas, dejando en evidencia la dificultad para configurar programas con especificidades disciplinares. El artículo concluye afirmando que este carácter transdisciplinar lleva a una diversidad metodológica y a una evanescente definición del objeto de estudio, que si bien no se constituyen en una desventaja epistemológica, prueban la falta de un elemento cohesionador e identitario para la disciplina.

\section{Palabras clave}

Ciencia política, epistemología, (trans)disciplinariedad, Colombia.

\section{Abstract}

The article proposes an epistemological interpretation of the disciplinary development of political science in Colombia. Through a hermeneutical exercise it builds the need to think and recognize the political science in a (trans) disciplinary way to show then that in the process of formation of the different faculties have participated professionals from different branches, revealing the difficulty to set up programs with disciplinary specificities. The article concludes that this transdisciplinary methodology leads to a diversity and an evanescent definition of the object of study, that while not constituting an epistemological disadvantage, prove the lack of a cohesive and identity element for the discipline.

\section{Keywords}

Political science, epistemology, (trans) disciplinarity, Colombia.

\footnotetext{
${ }^{1}$ Artículo del proyecto de investigación “¿Hacia dónde va la ciencia política en Colombia?" desarrollado por la Corporación Universitaria Autónoma del Cauca, Colombia; y del proyecto de investigación "Trayectorias de la ciencia política en Colombia: la influencia de los fenómenos políticos en el desarrollo disciplinar entre 1968 y 2012". Escuela de Política y Relaciones Internacionales de la Universidad Sergio Arboleda, Bogotá, Colombia.

${ }^{2}$ Doctor en Estudios Latinoamericanos, Universidad Nacional Autónoma de México. Candidato a doctor en Ciencias Sociales, Universidad Autónoma Metropolitana, México. Investigador asociado de la Corporación Universitaria Autónoma del Cauca, Popayán, Colombia.

Correo electrónico:

julian.caicedo@uniautonoma.edu.co

${ }^{3}$ Estudiante de doctorado en Estudios Políticos y Relaciones Internacionales de la Universidad Nacional de Colombia. Profesor de la Escuela de Política y Relaciones Internacionales de la Universidad Sergio Arboleda, Bogotá, Colombia; e investigador de la Corporación Universitaria Autónoma del Cauca, Popayán, Colombia.

Correo electrónico:

sergio.angel@usa.edu.co

${ }^{4}$ Politólogo, Universidad del Cauca, Colombia; especialista en Ciencia Política de la Universidad de IbaguéUniversidad de Salamanca; candidato a doctor en Ciencia Política de la Universidad de Belgrano, Buenos Aires. Profesor en la Universidad del Tolima, Ibagué, Colombia. Correo electrónico: jacuellara@ut.edu.co

Para citar este artículo: Caicedo, J., Angel, S., \& Cuellar, J. (2015). Una ciencia política transdisciplinar en Colombia. Revista Civilizar Ciencias Sociales y Humanas, 15(28), 135-150.
} 


\section{Introducción}

La pregunta sobre la manera como la ciencia política se constituyó como disciplina en nuestro país, pasa sin lugar a dudas, por el análisis y caracterización del proceso de institucionalización que da cuenta genealógicamente, del número de programas, instituciones, grados académicos oferentes, orientación investigativa, temas y/o núcleos de formación (DuqueDaza, 2013; Leyva et al., 2013; Parra, Tabares \& Hurtado, 2013). Sin embargo, tiene relación con otros elementos, medianamente referidos o estudiados a la hora de pensar la disciplina y debatir sobre su directriz: el modo en que las instituciones de educación superior entienden la profesión y toman la decisión de apertura a nuevos programas; las formas de enseñanza, es decir, la lógica interpretativa con la que se forma las generaciones de politólogos; el proceso de inserción laboral y la creación o existencia de un mercado laboral para los politólogos; y la discusión epistemológica, en términos de acuerdo o desacuerdo de paradigmas o enfoques, que atraviesa necesariamente cada uno de los aspectos en mención, y que permite otorgarle cierto factor de identidad a la disciplina.

Esto último alude a la generación del reconocimiento social de la necesidad técnica del profesional en ciencia política en el desempeño de algunas funciones en el sector privado y público: ¿qué politólogo estamos formando? Como señalan Bejarano y Wills (2005):

[...] la ciencia política, en estas décadas, ha pasado de defender causas políticas o paradigmas ideológicos, a comprender y explicar procesos para, a partir de ese conocimiento histórico, sugerir criterios o estrategias para la acción política. Estos autores han venido haciendo esfuerzos por dar cuenta de ese desarrollo disciplinar a partir de varios elementos, observando, entre otros, la profesionalización que evidencian los procesos de apertura de nuevos programas de pregrado y de posgrado, la producción bibliográfica, y la producción de los centros de investigación (p. 112).
El presente documento propone una interpretación sobre el carácter epistemológico de la ciencia política, planteando que es forzoso pensarla transversalmente, reconociendo paradigmas propios del ámbito de las ciencias sociales y de múltiples enfoques como aplicación de estos. Es decir que, proponemos abordar un cuarto elemento citado de forma contingente al sentido de la institucionalización: factor epistemológico, los otros tres son la docencia, la investigación y la comunidad académica (DuqueDaza, 2013), que consideramos indispensable para adentrarnos en la discusión de los demás elementos de la disciplina, que es válido decir, se ha visto "encasillada" en formas positivistas o neopositivistas de construcción y generación del conocimiento.

En este sentido, sugerimos "jugar" con el interrogante, retomando la pregunta de Sartori (2004) sobre la ciencia política estadounidense, ¿hacia dónde va (o debe ir) la ciencia política en Colombia? En términos generales, hablamos de una ciencia política comprometida con los sujetos y que forma sujetos comprometidos sociopolíticamente (la idea de vocación presente en Bulcourf \& Cruz, 2012) $)^{1}$. Sin embargo, nuestra respuesta es que la disciplina en Colombia debe ir hacia una transversalidad necesaria porque debe nutrirse de los paradigmas, enfoques, métodos y técnicas que permitan analizar, entender, interpretar, predecir, modelar, planificar, sostener y/o transformar la realidad política; y va hacia una transversalidad obligada porque los encargados de formar las generaciones de politólogos, responden en su mayoría, a orientaciones profesionales propias de las ciencias sociales, pero diferentes a la ciencia política.

La problematización en el caso colombiano no solo debe ser sobre quiénes hacen ciencia política, sino para qué y cómo, pasando de un factor descriptivo a varias líneas hipotéticas (propositivo), que lleven a pensar en muchas posibilidades legítimas y válidas de ciencia política, a la par que se construye desde el trabajo 
colectivo (asociaciones, organizaciones e instituciones) un escenario característico a la profesión, cohesionado y con una identidad.

En este orden de ideas, el presente artículo de reflexión se divide en dos partes: primera, se expone el debate epistemológico al interior de la ciencia política, dejando de presente la postura positivista de corte norteamericano y las posturas interdisciplinares o transdisciplinares que rompen con la noción de fronteras entre las diferentes ciencias sociales; y segunda, se alude al modo como se configuró la ciencia política en Colombia, revisando así el registro de los diferentes programas y su actual planta docente.

\section{El (des)acuerdo epistemológico}

La ciencia entendida como un conjunto sistemático de conocimientos, por lo visto encuentra en la política un "matrimonio (in) conveniente", un adjetivo que parece no pertenecerle y que en ocasiones hace dudar, legítimamente, de su sistematicidad (la forma de llegar a...). Hablamos de su carácter científico, porque el principio intersubjetivo de la forma de construcción del conocimiento político (que siempre es social como relación), parece falible (inexacto); de ahí la necesidad de modelarlo, proyectarlo, cuantificarlo y explicarlo a través de la "verdadera" manera de hacer ciencia, del único modo científico reconocido y válido, es decir, desde el paradigma positivista o neopositivista, que en muchas ocasiones implica generar "conocimiento a lo Rorty" esto es, pragmático y contrastable.

Miembros de la comunidad académica politológica colombiana han dado cuenta del desarrollo de la ciencia política en el país (Bejarano \& Wills, 2005; Leal, 1998; Losada, 2004; Murillo \& Ungar, 1999) y algunos otros trabajos descriptivos, complementarios, con base en los anteriores (Mejía-Quintana, 2006; Losada \& Casas, 2008 ; Cárdenas \& Suárez, 2010; Duque-Daza, 2013; Leyva et al, 2013; Angel \& Barrero, 2013, 2014; Caicedo \& Cuellar,
2015), abordan el problema desde múltiples sentidos: histórico-descriptivo, epistemológico, ontológico y metodológico; de una u otra forma se evidencia una preocupación por entender las propias dinámicas de construcción de la disciplina en el escenario colombiano, y aunque muchas veces evitando explicar su proceso como parte de un contexto latinoamericano en relación con el auge de las ciencias sociales, siempre ha estado presente el interrogante sobre la dirección que toma la ciencia política como ciencia y como disciplina. Es decir, la profesionalización desde su institucionalización $\mathrm{y}$ desde sus referentes epistemológicos.

Las disciplinas son producto de la profesionalización de una ciencia, lo que se demuestra en la institucionalización de la misma. Una disciplina académica se refiere a "un saber en cuyo nombre se expiden títulos académicos" (Losada, 2004, p. 10). Como consecuencia, una profesión se caracteriza por el control de un sistema de conocimiento abstracto del cual se derivan prácticas y técnicas (Abbot, 1988).

La ciencia política entendida como un conjunto de proposiciones generales sobre el mundo político, verificable empíricamente (Losada, 2004, p. 10), ha rechazado, por parte de un primer grupo que llamaremos fundacional, cualquier forma de construcción cognitiva que no sea pensada, referenciada o contrastada fácticamente y con una estructura cuantitativa angloamericana.

Consideramos que esto no permite que la disciplina en términos de comunidad, debates e identidad se consolide, al contrario, genera una situación de volatilidad y fragmentación de la comunidad politológica, y una especie de reconocimiento y exclusión de los nuevos programas, que se muestran distantes en contenidos, profesores, enfoques e incluso maneras de entender la disciplina. La idea de programas de ciencia política con escasos politólogos y en algunos casos sin ellos, como responsables en la formación, aparece como posibilidad. Es una obviedad entonces, 
las razones por las cuales la discusión epistemológica, no es siquiera tema secundario.

Para Bunge (2005, pp. 18-20) la ciencia desde un punto de vista estrictamente positivo y, por tanto, vigente en los años cuarenta y cincuenta, debe ser capaz de generar teorías explicativas generales y leyes que den cuenta de relaciones constantes entre variables, de modo que ayuden a trascender los hechos experienciales del observador. Esta idea de ciencia explica la necesidad de estudiar los fenómenos políticos a la luz de estructuras metodológicas fácticas y cuantitativas. Es decir, la necesidad de teorías explicativas y leyes en una realidad causal que en la búsqueda de legitimidad y validez ha distorsionado su espíritu y su compromiso, que debe ser ir más allá de explicar, y que le lleva a pensarse única y autónoma de una realidad fragmentaria llamada política, en contraste con una social, económica, cultural, histórica, etc.

Para Landreani (1990) en el caso de las ciencias sociales, y la ciencia política como una de ellas, la crisis del paradigma dominante parte del exacerbado empeño del positivismo de reducir la realidad social a través de un sistema de procedimientos de carácter cuantitativo. La crisis se manifiesta básicamente en la debilidad de los criterios de validez y confiabilidad positivistas que imponen al conocimiento científico frente a la complejidad y dinamismo de los fenómenos sociales, a pesar de los controles que en el ámbito de las técnicas se han planteado e implementado. Es la lógica de la reproducción del laboratorio para las ciencias sociales.

Para Sartori (2004, p. 350) primigeniamente la idea de una ciencia política en contraste con los estudios políticos o de gobierno, implicó la existencia de un lenguaje común y especializado entre la comunidad científica, donde los cimientos metodológicos y rigurosos eran la parte esencial del trabajo científico, pero nunca proyectada desde la amplia cuantificación metodológica en la que ha caído en años recientes.
Según Barrientos (2009) la crítica al cuantitativismo y la camisa de fuerza de la metodología politológica contemporánea nació en Estados Unidos. El libro coordinado por Kristen R. Monroe (2005) Perestroika!: the raucous rebellion in political science, recorre el debate metodológico al interior de la ciencia política y se propone alternativas sin perder la cientificidad. La crítica inicia exactamente con el famoso correo firmado por "Perestroika" (2000), quien señala sobre la American Political Science Review de la American Political Science Association:

¿Por qué todos los artículos de la APSR tienen la misma metodología -estadística o de teoría de juegos- en relación [con] un "simbólico" artículo de teoría política?... ¿Dónde está la historia política, la historia internacional, la sociología política, la metodología interpretativa, el constructivismo, los estudios de área, la teoría crítica y por qué no, el posmodernismo? (p. 45).

El argumento central de los autores es simple: la ciencia política contemporánea, aquella de los journals norteamericanos, no puede darse aires de estar científicamente por encima de la política.

En este sentido, consideramos como planteamiento, que la discusión sobre la orientación de la ciencia, en este caso política, debe partir del sentido de los paradigmas y la manera, como señala Roth (2008, p. 69), en la que se busca capturar la realidad mediante la elaboración de teorías que permitan explicarla, describirla, predecirla, interpretarla o transformarla (de forma total o en partes), a través de la selección de los factores o variables considerados como determinantes. Es el debate que se debe redirigir hacia el modo de hacer ciencia de la política y para la política.

Pensarla desde los paradigmas (en plural) nos puede permitir encontrar caminos o aventurar propuestas sobre una ciencia política adjetivada desde lo territorial (caracterización 
del fenómeno de la política en sí: latinoamericana, europea, colombiana, argentina, estadounidense, etc.); o desde la interacción del sentido ontológico, epistemológico y metodológico (disciplinar, multidisciplinar, interdisciplinar, transdisciplinar $)^{2}$.

Kuhn (1974) sostiene que las ciencias particulares crean paradigmas -grandes teorías en general aceptadas por la comunidad científica- con los cuales se desarrolla lo que llama "ciencia normal". Las revoluciones científicas se producen cuando se van descubriendo nuevos hechos que no encajan dentro de los moldes del paradigma establecido. Sin embargo, las ciencias sociales, por no tener un paradigma generalmente aceptado que guíe sus esfuerzos en pro del conocimiento, no son más que una "protociencia", de ahí la ciega "necedad" por adoptar marcos explicativos que legitimen, a modo de comunidad científica, el conocimiento político o de lo político desde la disciplina, entendida como antítesis de la ciencia (normal).

Parafraseando a Strasser (1979, p. 16), así se escribe la ciencia por nosotros y entre nosotros. Y así se reniega (explícitamente) de algunas tradiciones tanto como de algunas posibilidades y necesidades. En muchas ocasiones obviamos el para qué, o no se sabe muy bien qué, o no se sabe muy bien a cambio de qué. Esta necesidad de que la ciencia sea ciencia desde el paradigma dominante, que a pesar de cierta pertinencia no puede pensarse como único, ha llevado a la disciplina hacia el camino de la crisis, por su excesiva cuantificación metodológica (Sartori, 2004), incluso agregaríamos, porque los métodos positivistas le han hecho perder de vista la complejidad social.

Por ello, hablar de ciencia política debe implicar un análisis de al menos cuatro líneas hipotéticas: a) la ciencia política es una disciplina joven cuya formalidad data de la revolución behaviorista y conlleva la separación de hechos y valores y la adopción necesaria de una estructura metodológica, ontológica y epistemológica propia de las ciencias naturales (hipótesis fundacional); b) la ciencia política involucra un estudio del pensamiento político, enfatizando en las formas y sentidos de la evolución de las ideas, esto es, pensada desde la teoría política, donde ciencia y filosofía se contienen desde una forma contrafáctica y la estructura metodológica, ontológica y epistemológica responde a un principio heurístico (hipótesis histórico-interpretativa); c) la ciencia política debe dirigirse a procesos de transformación social a través de la acción-participación y de valores, porque la política está en todas partes y la objetividad es una ilusión (hipótesis crítica); y d) la ciencia política debe comprenderse más allá de los especialistas, en tanto que la política es una manifestación del pensamiento que no puede ser alejada de los valores y las formas enriquecedoras de la imagen, la letra y el espíritu (Alarcón, 2010, p. 41), nos referimos a una ciencia transversal, transdisciplinar que no abandona su identidad pero que permite una conjunción con otros saberes, con otras formas de conocimiento, de ciencia, y no solo explica sino que además interpreta y transforma porque parte de la idea de que la realidad es compleja (hipótesis metapolítica).

Estas líneas hipotéticas, ninguna de ellas descartable por cierto, descansan sobre cuatro paradigmas reconocibles en las ciencias sociales: (neo)positivista; pospositivista o racionalismo crítico; crítico o de la teoría crítica; y constructivista o hermenéutico, diferenciables, como señala Guba (1990, p.18), por las respuestas que se dan a las tres preguntas fundamentales: a) pregunta ontológica: atañe a la forma y naturaleza de la realidad y, por tanto, a lo que es posible conocer; b) pregunta epistemológica: que incumbe a la naturaleza de la relación entre el sujeto cognoscente y el objeto que puede ser conocido y, en consecuencia, a la posición que debe asumir el investigador respecto a su objeto de conocimiento $\mathrm{y}, \mathrm{c}$ ) pregunta metodológica: que se refiere a la forma como el investigador puede proceder para hallar el conocimiento de aquella realidad que cree que puede ser conocida. 
Para Resnik (1998, p. 32) en el campo de la ciencia política, como parte de las ciencias sociales, son evidentes cinco paradigmas: marxista o marxiano, fisicalista, weberiano, habermasiano y sistémico. Así mismo es posible clasificar desde un punto de vista metodológico la producción académica (investigaciones) (Mertz, 1984) en tres categorías: teoría política normativa; análisis institucional y empirismo científico, y son útiles porque señalan múltiples formas de analizar los fenómenos políticos (enfoques), y porque identificados históricamente, pueden significar una fiel descripción del desarrollo de la ciencia política.

Es claro que dependiendo de la visión o "necesidad" del investigador por interpretar el mundo, se aboga por teorías diferentes, en ocasiones equidistantes entre ellas. Es decir, existen múltiples estrategias de explicación, diversas explicaciones o interpretaciones de los fenómenos a analizar y muchos sentidos de lo que se asume y discierne como paradigma en la construcción del conocimiento. Para Roth (2008) la teoría es como un mapa con el cual se pretende simplificar la realidad para hacerla entendible. Debemos comprenderla como una reducción extrema de la complejidad del mundo, aunque recordemos que ninguna teoría por sí misma es capaz de dar cuenta de esta complejidad de manera unívoca "científicamente" correcta.

En este punto, es menester diferenciar un enfoque de una teoría y de un modelo, de tal suerte que el uso responda epistemológicamente a un modo asertivo de construcción del conocimiento, sin descalificar métodos, técnicas o la lógica en la sistematización de los mismos. Para Balme y Brouard (2005), la "asociación y manera de considerar los problemas, un repertorio metodológico y un vocabulario interpretativo" (pp. 35-36), se puede denominar enfoque (marco), que parte de un constructo teórico y a su vez de modelos establecidos de allegar a la realidad definiendo su sentido.

Asíuna teoría es "una serie de proposiciones ligadas lógicamente entre ellas que buscan explicar una serie de fenómenos" (Sabatier, 1999, p. 261). Puede considerarse como parte de un sistema lógico cuyas observaciones, axiomas, postulados y proposiciones procuran señalar las condiciones de desarrollo de ciertos supuestos siendo posible especular, deducir, interpretar y/o postular mediante ciertas reglas o razonamientos.

Podemos identificar cuatro tipologías: a) descriptiva, referente a características específicas de personas, grupos, situaciones o acontecimientos; b) explicativa, indica las relaciones entre diversos fenómenos; c) predictiva, fija tipos concretos de relaciones entre fenómenos y constructos particulares; y d) normativa, que compete a los fenómenos poniendo el acento en lo que puede o debe ser la realidad adjetivada o genérica.

Por último, para Ostrom un modelo es "representación simplificada de un proceso (puede ser parte de una teoría)" (citado por Sabatier, 1999, p. 262) que "precisa hipótesis en relación [con] un número limitado de parámetros y variables". De ahí que un modelo se inscriba en una teoría y esta, en conjunto con otras teorías, forma parte de un enfoque de análisis. El marco es indispensable porque permite la selección de variables, hipótesis, indicadores y relaciones causales, básicamente desde un sentido hipotético-deductivo de la investigación o construcción del conocimiento, que empero, no es la única manera de llegar a este (Roth, 2008).

Al respecto, en Losada y Casas (2008) se evidencia un posicionamiento de superación del debate sobre los macromoldes (paradigmas), incluso definiendo el carácter de cientificidad de la politología, en tanto que tiene un referente empírico, y se plantea una propuesta ampliamente descriptiva de 21 enfoques para el análisis político, casi a modo de hoja de cotejo con la producción intelectual, mayormente anglosajona.

Tengamos en cuenta que Hay (2002, p. 11) habla de corrientes: viejo y nuevo institu- 
cionalismo, conductismo y posconductismo, y teoría de elección racional; Pierson y Skocpol (2008, pp. 7-38) de paradigmas de análisis en la ciencia política: el racional, el del institucionalismo y el conductista; Alarcón (2010, p. 36) sobre la base del trabajo de Hay, propone cinco enfoques: viejo y nuevo institucionalismo, conductismo y posconductismo, elección racional, marxismo y posmodernismo.

Es válido decir que aunque la propuesta de Losada y Casas cae en repeticiones y algunas omisiones sobre todo en el campo de la hermenéutica y de los estudios latinoamericanos, es importante reconocerla como el primer ejercicio en Colombia que se atreve a ampliar el marco de análisis a nuevas lógicas de construcción de conocimiento sociopolítico, y aunque es clara la adherencia de los autores al modelo (neo)positivista, los demás lineamientos que plantean son herramientas que pueden definir la ruta del quehacer politológico en aras de reconocer, muy a pesar de los autores, un escenario de heterogeneidad con una ciencia política transversal, transdisciplinar, y no solamente una ciencia política que se orienta y devela desde la explicación y la comprensión de la realidad política (Losada \& Casas, 2008, p. 297). En armonía con Masías (2009) en relación con la controversia epistemológica referida en Losada y Casas (2008),

[...] en Colombia, donde hay "ciencia política" y "estudios políticos", al tiempo que hay "analistas políticos" y "periodistas políticos", y donde, para hacer todavía más compleja la discusión, hay auspicio de otras formas de conocer diferentes a la científica dentro de la academia, que no necesariamente coinciden con los rótulos anteriores, una controversia como la suscitada por el texto es de suma utilidad y gran relevancia (p. 308).

¿Enfoques, teorías o modelos? En la actualidad el conocimiento no puede imponer reglas, no debe imponerlas, aunque sí, respetar criterios epistemológicos. Debe permitirse enfrentar la realidad, política en este caso, de una manera desideologizada, sin la preocupación de validez sino de legitimidad que tanto constriñe a la comunidad académica de hoy por las instituciones encargadas de financiar la investigación y la producción académica. No puede darse equivocadamente la "línea" de trabajar o formar desde cierto enfoque, modelo, técnicas, marco o métodos, para generar verificación o comprobación empírica de un hecho concreto, ya que en un escenario de totalidad y complejidad, lo subjetivo (sujeto) y abstracto (simplificado a un contexto), son los elementos de la realidad social y no se pueden ignorar.

\section{Hacia una ciencia política transversal}

Queda en evidencia en muchos escenarios académicos y en la forma de construcción del conocimiento, que el camino a seguir por la ciencia política en Colombia, siempre ha sido claro: reproducir el carácter epistemológico de la ciencia política estadounidense, no solo enfatizando en el estudio del sistema, sino en su orientación metodológica cuantitativa, de modelación y comparativista.

Hoy, más de cuarenta años después está vigente lo descrito por Sorauf y Hyneman (1967, p. 11) sobre la ciencia política estadounidense; es decir, el interés por estudiar los procesos, el comportamiento y las instituciones de los sistemas políticos, con miras a formular generalizaciones y explicaciones sistemáticas acerca de lo político; buscar generalizaciones acerca de las relaciones entre los sistemas políticos, en particular la política interna dentro del sistema internacional; analizar el producto final, los planes públicos del proceso político; y examinar ideas y doctrinas acerca del gobierno y del sistema político, ideas tales como los conceptos y justificaciones de la democracia, la justicia y la igualdad.

Consideramos, siguiendo a Emmerich (1997), que los intentos por justificar la existencia de múltiples ciencias sociales "recortando" algún aspecto de la realidad social y declarán- 
dolo objeto específico de una u otra disciplina, se basan en una concepción de la sociedad y la acción social según la cual los hechos o fenómenos sociales tendrían rasgos característicos que permitirían clasificarlos como hechos eminentemente políticos (v. g. un acto electoral), eminentemente jurídicos (v. g. la sanción o aplicación de una ley) y eminentemente económicos (v. g. la producción de algún bien). Es decir, la sociedad tendría una esfera propiamente política, otra esfera económica, otra cultural, y así sucesivamente.

Entonces, las ciencias sociales particulares tendrían por objeto concreto alguna de estas esferas de la sociedad. Las diferencias y taxonomías epistemológicas así han funcionado. No obstante, la transversalidad cognitiva de las disciplinas haría posible sobreponerse a dicha fragmentación de lo social, y nos llevaría a una "reconciliación" con la idea fundacional de las ciencias sociales, esto es, la posibilidad de construir un conocimiento que no esté adjetivado y sea pensado en términos de un proceso de interacción y relación de una sociedad, donde nos acerquemos a la expresión filosófica que no puede separarse de la ciencia en estricto sentido. Una metapolítica implicaría una crítica que sea capaz de aparecer en el espíritu de nuestra política, como acción, como proceso.

¿Existe algo o algún lugar donde no haya política? Porqué reducir la posibilidad de su estudio a un tema o a un proceso. Tenemos que reflexionar no solo desde la modernidad, sino desde el fenómeno político que se remite a los clásicos del pensamiento político occidental: de Aristóteles a Platón, Maquiavelo, Hobbes, Locke, Marx, y también latinoamericano: Zea, Salazar Bondi, Martí, Dussel, Sánchez Vázquez y Bolívar Echeverría. Sería pensar una ciencia política que no se limite a estudiar el comportamiento "observable" de los actores sociales y el funcionamiento de los sistemas políticos (contemporáneos), sino que además, analice los medios, los fines y el "sentido" de la experiencia política (e incluso, en un nivel ulterior de reflexión, los medios, los fines y el "sentido" de la propia indagación sobre la experiencia política) (Zolo, 1989, p. 45).

Por ello una ciencia política transversal, no es otra cosa que la inserción de la disciplina en lo transdisciplinar. Lo multidisciplinar lo entendemos como un proceso de convergencia de varias disciplinas diferentes, hacia el abordaje de un mismo problema o situación, no existe una relación aparente entre los contenidos. Lo interdisciplinar como una interacción entre dos o más disciplinas que puede ir desde la simple relación de ideas hasta la integración recíproca de conceptos fundamentales. Y lo transdisciplinar lo asumimos como un pensamiento de un nuevo orden que posibilita un nuevo espacio para el saber. Requiere de la construcción de conocimientos basados en la interrelación de las diversas complejidades, niveles y contextos, donde el sujeto que participa activamente, se piensa articulando el todo con cada una de sus partes. La identidad disciplinar se mantiene aunque el proceso de interrelación sustituye sus límites. El diferenciador no es el proceso de formación cognitiva, sino la acción concreta que plantea a manera de división social del trabajo (necesaria), una posibilidad no de segregación y exclusión, sino de reconocimiento profesional. Como señala Alarcón (2010):

[...] no se trata de que el politólogo sea un aprendiz de todo y un experto en nada. Sin embargo [...] un experto en la problemática política puede seguir teniendo bases generales de teoría, metodología y técnicas prácticas que le permitan abordar cualquier dimensión problemática en la que se encuentre inserto (p. 33).

Hablamos entonces, según Emmerich y Alarcón (2007), de una ciencia política al servicio de los ciudadanos, una ciencia política al servicio de los sujetos, una ciencia política transdisciplinar, transversal.

Alarcón (2010, p. 39) traza un vínculo interdisciplinario entre la ciencia política y nueve disciplinas adjetivadas políticamente: antropo- 
logía, filosofía, sociología, administración y políticas públicas, geografía y política comparada, economía, historia, psicología y derecho político y constitucional. Sin embargo, el elemento relacional que propone, es transdisciplinar en términos de una empatía con objetos de estudio y formas de acercarse a la realidad política.

Más adelante evidenciaría esta situación al proponer que debe hacerse comprender más allá de los especialistas, de manera que el estudio se lea y vea desde el ámbito literario, cinematográfico y visual, de tal suerte que aprendamos a "sentir y disfrutar la política" (p. 40).

Cabe recordar que los padres fundadores no se preocuparon mayormente por ser llamados sociólogos, politólogos o economistas: eran científicos sociales integrales, científicos de la sociedad en su compleja totalidad (Emmerich, 1997). Duverger (1988) relata que:

Augusto Comte y los sociólogos franceses de fines del siglo XIX, especialmente Durkheim, eran contrarios a esta dispersión y cita a Comte cuando el creador del término sociología afirmaba que los fenómenos sociales son profundamente conexos (pp. 29-30).

Y que todo estudio de una categoría parcial de ellos resulta estéril. De ahí que la ciencia política pueda entenderse desde su quehacer como una disciplina forzosamente transversal, en aras de reivindicar el carácter del conocimiento desde el principio relacional de la complejidad y la totalidad. La máxima aristotélica puede tener un mayor sentido, podemos estudiar la parte, solo que no olvidemos que antes de la parte está el todo.

\section{El escenario colombiano}

El proceso de institucionalización de la ciencia política en Colombia, inició con la creación del programa de Ciencia Política de la Universidad de los Andes en 1968. Cuatro años después la Pontificia Universidad Javeriana funda la maestría en Estudios Políticos, y en 1975 se instaura la maestría en Ciencia Política en la Universidad de los Andes (Duque-Daza, 2013).

En la década de los setenta, siguiendo a Murillo y Ungar (1999), se constatan dos tendencias dentro de la disciplina; por un lado, el desarrollo de estudios estrictamente politológicos, y por el otro, el de estudios de corte interdisciplinario, como los realizados por el Centro de Investigación y Educación Popular (Cinep) y el Instituto de Estudios Políticos y Relaciones Internacionales (IEPRI) de la Universidad Nacional de Colombia. Es durante estos años que se abren nuevos programas de posgrado, como la maestría en Estudios Políticos de la Pontificia Universidad Javeriana en 1972, y la maestría en Ciencia Política de la Universidad de los Andes, en 1975.

Si bien los setenta y ochenta no se caracterizaron por la apertura de nuevos programas de pregrado, sí aparece un grupo de publicaciones que da cuenta de las investigaciones de tipo político en diferentes universidades e institutos, destacan la revista Ciencia Política del Instituto de Ciencia Política de Bogotá, la revista Foro, de la Fundación Foro Nacional por Colombia, y la revista Análisis Político del IEPRI.

En la década de los noventa despega el proceso de institucionalización con la creación de nuevos programas de pregrado. En 1994 se abre el programa de Ciencia Política en la Universidad Nacional de Colombia en su sede de Bogotá, continúa en 1995 con la apertura de los programas de la Universidad Nacional de Medellín, la Pontificia Universidad Javeriana de Bogotá y la Universidad Externado de Colombia. En 1996 se inaugura el programa de la Universidad del Cauca y en 1998 el programa de Estudios Políticos y Resolución de Conflictos en la Universidad del Valle. Así, durante la década de los noventa se crean seis nuevos programas de pregrado, dos décadas después de que se erigió el primer programa de pregrado y los primeros posgrados. 
Pero es en los últimos trece años donde florece la oferta de pregrados sobre la disciplina. Entre 2000 y 2013, 25 nuevos programas de pregrado en Ciencia Política fueron ofertados, nueve de ellos a partir de 2010. Igualmente, durante los últimos treinta años son numerosos los programas de posgrado en Ciencia Política o afines que han sido ofrecidos. Estas cifras nos indican que el proceso de profesionalización de la ciencia política en Colombia está apenas en consolidación.

Dos escenarios se han definido desde entonces: por una parte, los primeros politólogos fueron escasos; por otra, los investigadores políticos mantienen una tendencia; poseen una formaciónenotras disciplinas comola sociología, historia, derecho, filosofía y más recientemente administración pública, que se refleja en los múltiples estudios interdisciplinarios de la década de los setenta. Los politólogos formados en pregrado empiezan a crecer en número solo a partir del año 2000 aproximadamente, cuando comienzan a egresar las primeras cohortes de los programas creados a partir de 1995. Pero es en los próximos años que la cifra de politólogos va a aumentar de manera considerable, dado el guarismo de nuevos programas abiertos.

Sin embargo, esta explosión de programas de ciencia política suscita muchos interrogantes, ya planteados incluso desde los noventa. ¿Existe la planta de profesores calificada para formar a las nuevas generaciones de politólogos?
¿Qué orientación profesional tienen las nuevas cohortes de politólogos egresados?

La centralización de los programas es otra variable sobre la que se merece analizar dicho crecimiento, ya que los nuevos programas ofertados en ciudades principales como Bogotá (incluido el municipio de Chía donde opera la Universidad de La Sabana), Medellín, Cali y Barranquilla, sobre la base de 32 programas, concentran 24, es decir, el $75 \%$. Esto ocasiona que programas regionales en ciudades intermedias y pequeñas, experimenten problemas para conformar una planta docente especializada en el área, y en algunos casos, el número de estudiantes es reducido, pues estos se interesan por ubicarse en ciudades principales, aunque sean programas de reciente creación, que "garantizan" una formación especializada.

La tabla 1 muestra en orden cronológico las fechas de creación de los diferentes programas y su orientación, tomando como referencia la formación profesional de los profesores de planta. En la exploración, nos encontramos con programas con orientación politológica, cuando los educadores de tiempo completo del programa, son politólogos de formación y se garantiza una o varias líneas de formación; transversal, cuando menos del $50 \%$ de docentes de planta, tiene una formación profesional en otras áreas del conocimiento (historia, sociología, derecho, filosofía, administración y relaciones internacionales).

Tabla 1.

Programas de ciencia política en Colombia

\begin{tabular}{|c|c|c|c|}
\hline Institución & Programa & Año & Orientación \\
\hline 1. Universidad de los Andes & Ciencia Política & 1968 & Politológica \\
\hline 2. Universidad Nacional de Colombia (Bogotá) & Ciencia Política & 1994 & Politológica \\
\hline 3. Universidad Nacional de Colombia (Medellín) & Ciencia Política & 1995 & Politológica \\
\hline 4. Pontificia Universidad Javeriana (Bogotá) & Ciencia Política & 1995 & Politológica \\
\hline 5. Universidad Externado de Colombia & Gobierno y Relaciones Internacionales & 1995 & Transversal \\
\hline
\end{tabular}




\begin{tabular}{|c|c|c|c|}
\hline Institución & Programa & Año & Orientación \\
\hline Universidad del Cauca & Ciencia Política & 1996 & Transversal \\
\hline Universidad del Rosario & Ciencia Política y Gobierno & 1996 & Transversal \\
\hline Universidad del Valle & Estudios Políticos y Resolución de Conflictos & 1998 & Transversal \\
\hline Pontificia Universidad Javeriana (Cali) & Ciencia Política & 2001 & Politológica \\
\hline Universidad Tecnológica de Bolívar & Ciencia Política y Relaciones Internacionales & 2001 & Transversal \\
\hline Universidad Militar Nueva Granada & Relaciones Internacionales y Estudios Políticos & 2001 & Transversal \\
\hline Universidad de Antioquia & Ciencia Política & 2003 & Politológica \\
\hline Universidad Eafit & Ciencias Políticas & 2003 & Transversal \\
\hline Universidad Pontificia Bolivariana & Ciencias Políticas & 2003 & Transversal \\
\hline Universidad Icesi & Ciencia Política & 2004 & Politológica \\
\hline Universidad Sergio Arboleda & Política y Relaciones Internacionales & 2004 & Transversal \\
\hline Universidad de San Buenaventura (Bogotá) & Ciencia Política & 2005 & Transversal \\
\hline Universidad de Ibagué & Ciencia Política & 2006 & Transversal \\
\hline Universidad Autónoma de Manizales & Ciencias Políticas, Gobierno y Relaciones Internacionales & 2008 & Transversal \\
\hline Fundación Universidad del Norte & Ciencia Política y Gobierno & 2008 & Politológica \\
\hline Universidad Mariana & Ciencia Política & 2009 & Transversal \\
\hline Universidad del Tolima & Ciencia Política & 2009 & Transversal \\
\hline Fundación Universitaria Cervantina San Agustín & Ciencia Política & 2010 & Transversal \\
\hline Universidad de San Buenaventura (Cali) & Gobierno y Relaciones Internacionales & 2010 & Transversal \\
\hline Fundación Universitaria del Área Andina & Ciencias Políticas & 2011 & Transversal \\
\hline Politécnico Grancolombiano & Ciencia Política & 2011 & Transversal \\
\hline Universidad Santo Tomás & Gobierno y Relaciones Internacionales & 2011 & Transversal \\
\hline Universidad Surcolombiana & Ciencia Política & 2011 & Transversal \\
\hline $\begin{array}{l}\text { Fundación Universidad de Bogotá Jorge Tadeo } \\
\text { Lozano }\end{array}$ & Ciencia Política y Gobierno & 2011 & Politológica \\
\hline Universidad de La Sabana & Ciencias Políticas & 2012 & Transversal \\
\hline Universidad Autónoma del Caribe & Ciencias Políticas & 2012 & Transversal \\
\hline Universidad El Bosque & Ciencia Política & 2013 & Transversal \\
\hline
\end{tabular}

Fuente: elaboración propia con base en los datos del Ministerio de Educación Nacional (2015), e información obtenida en páginas de los distintos programas relacionada con profesores de planta.

En esta exploración, encontramos que solamente en nueve programas de pregrado existe más del $50 \%$ de profesores con formación en la disciplina, en las 23 restantes la formación recae en profesionales de diferentes disciplinas, principalmente derecho, sociología y relaciones 
internacionales. Dos lecturas y posiciones aparecen. La primera, evidentemente la comunidad académica reconoce la labor de los profesionales que aun no siendo politólogos de base se han comprometido con los estudios políticos y se dedican a ellos de modo permanente.

No obstante, es necesario analizar las herramientas epistemológicas, metodológicas y conceptuales que pueden aportar, por ejemplo, sociólogos, antropólogos, economistas, historiadores, economistas, abogados y administradores, que desde disciplinas sociales hermanas, pueden enriquecer los contenidos curriculares de las diversas carreras de ciencia(s) política(s) y adyacentes, pero ¿las bases epistemológicas y metodológicas permiten identificar y abordar un objeto de estudio de la misma manera?

No negamos que las especializaciones de posgrado permiten robustecer marcos teóricos y metodológicos de la disciplina, por ello nos referimos a la orientación profesional en pregrado, empero, es importante aclarar, como señala Angel y Barrero (2013), que no existen métodos exclusivos de la ciencia política en la praxis profesional colombiana. En una investigación sobre el tema, los autores en mención concluyen que los distintos programas de ciencia(s) política(s), se abocan a temas y métodos propios de las ciencias sociales, pero no es claro un elemento diferenciador para la disciplina. Habría que preguntar entonces, si el transversalismo que caracteriza la oferta de pregrados en Colombia es el causante de dicha diversidad. Es decir, tenemos un escenario de politólogos formados en muchas instituciones, desde disciplinas adyacentes y sin un elemento diferenciador.

Dicho lo anterior, es preciso agregar que los programas de ciencia política en Colombia no reciben una denominación unívoca, sino que por el contrario responden a intereses, lógicas, propósitos e iniciativas disímiles, pero si todos ellos son catalogados como parte de una sola disciplina, ¿a qué se debe la heterogeneidad en la nomenclatura?
En otro escrito Angel y Barrero, (2014) afirman que la razón de fondo para hablar de diferentes programas es el marketing educativo, de tal suerte que los programas para ser más atractivos dentro de la amplia oferta nacional incluyen determinados apellidos. Para otros (Leyva et al., 2013) esta nomenclatura, más allá de las razones de mercadeo, tiene un impacto sobre la praxis educativa, de tal modo que los programas con un apellido en relaciones internacionales, tendrán un mayor énfasis en esta que en otras áreas. De cualquier manera, en la tabla 1 se incluyen todos aquellos programas que a pesar de su nombre pueden entrar en la nomenclatura de la ciencia política, descartando así programas exclusivos de relaciones internacionales o de administración pública. Ahora bien, en contraposición con este planteamiento, Parra et al. (2013) consideran que

[...] en un mundo académico altamente especializado como el de hoy, [...] la re-hechura del tejido disciplinar dirige la mirada hacia la interdisciplinariedad y la transdisciplinariedad, en la que construir conocimiento político deja de ser un acto solitario y parcelado para constituirse en el resultado del trabajo colaborativo y complementario entre investigadores interesados en el mundo de la política (p. 33).

A esto le denominan "enfoque integrador", que se asocia a la posibilidad de construir nuevos marcos epistemológicos y teóricos desde la ciencia política, para acercarnos investigativamente a nuevos escenarios de compleja envergadura (como el latinoamericano) y propiciar un proceso de descolonización. Este enfoque solo es posible con la anuencia de investigadores formados en otras áreas del conocimiento o disciplinas.

En cualquiera de los dos sentidos, vale la pena mencionar que un avance muy importante sobre este debate fue la creación de la Asociación Colombiana de Ciencia Política (ACCPOL) en el 2006. Con la ACCPOL se demuestra que la comunidad académica nacional está en capacidad de reflexionar respecto de su propio 
desarrollo, identificar sus retos y plantearse objetivos comunes, es un paso significativo para el afianzamiento de la disciplina en el país. De hecho, los últimos esfuerzos de la asociación se han dado en esa dirección autorreflexiva, aunque no cuenta aún con participación masiva e integración que se necesitan para consolidar la disciplina, contrario a los diversos señalamientos (Leyva et al., 2013).

Por último, aunque son muchos los retos, vale la pena enunciar dos: primero, fortalecer una comunidad académica cohesionada, activa, reflexiva y con una identidad; y segundo, con el liderazgo de la ACCPOL, proponer y desarrollar lecturas y debates sobre las ventajas, alcances, límites y limitaciones de que la ciencia política en Colombia se dirija hacia una transversalidad.

\section{Consideraciones finales}

Intentamos abordar el tema de la transversalidad de la ciencia política desde dos ópticas. La primera, desde el factor epistemológico, indicando la necesidad de que se piense la disciplina dentro de los criterios metodológicos propios de las ciencias sociales, permitiendo una formación integral en los distintos paradigmas, teorías, enfoques, etc., dejando de lado el discurso de la "pureza" del método y de la disciplina.

La idea es propiciar una ciencia política comprometida con los sujetos sociales que explica, describe, predice, analiza, modela y/o proyecta la realidad política (social y del poder). El objeto de estudio es claramente identificado en las dinámicas del sistema, el Estado, el poder político y la democracia (Mejía-Quintana, 2006), así como en la intersubjetividad (entre sujetos).

Por otra parte, proponemos dejar sobre la mesa y para la discusión, el tema de la orientación transversal de la disciplina, que nos presenta un escenario disciplinar complejo que no es potestad de politólogos. Nuestra aseveración se refuerza con la revisión de la planta docente en cada una de las universidades que oferta el programa, y con la comprobación de que la orientación profesional que menos aparece, paradójicamente, es la de politólogo.

Advertimos entonces, que el problema de diversidad metodológica, de objeto de estudio y la falta de un elemento cohesionador e identitario, se asociarían a dicha situación. Muy pocas instituciones con programas de reciente creación, apuestan por politólogos con formación de posgrado para su planta profesoral.

Así mismo, siguiendo a Duque-Daza (2013), son identificables tres etapas del proceso de institucionalización

[...] la primera (1968-1989) corresponde a los inicios y a una débil institucionalización, además de su concentración exclusiva en Bogotá y en universidades privadas; la segunda (19892000) corresponde a una expansión gradual, pero con alta concentración y debilidades estructurales de la disciplina; y la tercera (20012012) es el de la expansión y un avance en su institucionalización con situaciones diversas y grados diferentes de desarrollo según el tipo de universidades y los soportes organizativos con que cuente (pp. 15-16).

Sin embargo, existe una "coyuntura crítica", que hace necesaria una cuarta etapa ligada con la creación de la ACCPOL, que debe iniciar un proceso de acercamiento de las formas de construir la disciplina en las instituciones educativas, sin utilizar un criterio de regulación que homogeneice el quehacer politológico, pero sí que le aporte elementos articuladores y una visión en términos profesionalizantes.

\section{Notas}

${ }^{1} \mathrm{Al}$ respecto, no hacemos referencia a un compromiso ideológico o filiacionista de los politólogos con ciertas instituciones, sino a un compromiso ético profesional, que puede tener diversas orientaciones: cambio, transformación, 
adaptación, etc., al entorno o a las circunstancias del escenario colombiano. Weber remite al "vivir de la política" y "vivir para la política"; apuntamos a esta segunda enunciación.

${ }^{2}$ Aclaremos que, como señala Emmerich (1997), en las ciencias sociales no hay formas generalmente aceptadas de concebir y estudiar la realidad. Por el contrario, existe una variedad de orientaciones teórico-metodológicas en competencia, nacida de la pluralidad misma de las sociedades humanas y de las opiniones que los hombres (incluidos los propios científicos sociales) tienen sobre ellas (c.f. Emmerich, 1997).

\section{Referencias}

Abbot, A. (1988). The System of Professions: An Essay on the Division of Expert Labor. Chicago: The University of Chicago Press.

Alarcón, V. (2010). “Ciencia Política”. En V. Martínez \& E. Villareal (Coords.), (pre) textos para el análisis político (pp.2348). México: FLACSO.

Angel, S., \& Barrero, F. (2013). La ciencia política y sus métodos: una comparación de los programas de ciencias sociales en Colombia. Co-herencia, Vol. 10(19), 207-234.

Angel, S., \& Barrero, F. (2014). Los retos de la Ciencia Política en Colombia. Revista Iberoamericana América Latina-EspañaPortugal, 14(56), 191-195.

Balme, R., \& Brouard, S. (2005). L'analyse politique de l'action publique: confrontation des approches, des concepts et des méthodes. Revue Française de Sciences Politiques, 55(1), 33-50.

Barrientos, F. (2009). Los desvaríos de un ex-politólogo: César Cansino y «La "muerte" de la Ciencia Política. Recu- perado de http://criticacida.wordpress. com/2009/03/02/hello-world/

Bejarano, A., \& Wills, M. (2005). La ciencia política en Colombia: de vocación a disciplina. Revista de Ciencia Política, 25(1), 111-123.

Bulcourf, P., \& Cruz, P. (2012). La ciencia política como profesión. Revista POSTData. Recuperado de https://docs.google.com/ file/d/0B637k_n5waBpOTUwMWRjZDktMWExMy00OTZmLWE0NzctYTQ0NTliMjgxNGQ0/edit?usp=drive web\&urp=http://www.revistapostdata. com.ar/2012/01/la-cienc\&pli=1

Bunge, M. (2005). La ciencia. Su método y su filosofia. México: Suramericana- Laetoli.

Caicedo, J. \& Cuellar, J. (Comp.) (2015) ¿Hacia dónde va la Ciencia Política? Algunas reflexiones sobre la disciplina en Colombia. Ibagué: Unitolima.

Cárdenas, F., \& Suárez, L. (2010) La ciencia política, ciencia noética del orden. Colombia Internacional, 72, 111-132.

Duque-Daza, J. (2013). Tres momentos de la institucionalización de la enseñanza de la Ciencia Política en Colombia 1968-2012. En S. Leyva (Ed.), La ciencia política en Colombia: ¿una disciplina en institucionalización?. (pp. 57-100). Medellín: EAFIT; ACCPOL.

Duverger, M. (1988). Métodos en las ciencias sociales. Barcelona: Ariel.

Emmerich, G. (1997). Metodología de la Ciencia Política. México: UAM.

Emmerich, G., \& Alarcón, V. (2007). Tratado de Ciencia Politica. México: Anthropos; UAM. 
Guba, E. (Ed.) (1990). The paradigm dialog. Londres: Sage.

Hay, C. (2002). Political Analysis: A Critical Introduction. Basingstoke: Palgrave.

Kuhn, T. (1974). La estructura de las revoluciones cientificas. México: FCE.

Landreani, N. (1990). Métodos cuantitativos versus métodos cualitativos: Un falso dilema. Ciencia, Docencia y Tecnología, 13(25), 17-39

Leal, F. (1988). La profesionalización de los estudios políticos en Colombia. Análisis Político, 3, 49-62.

Leyva, S. (Ed.), Muñoz, P., Fortou, J., Preciado, A., Ramírez, M., Daza, J., \& Murillo, G. (2013). La ciencia política en Colombia: ¿una disciplina en institucionalización?. Medellín: EAFIT; ACCPOL.

Losada, R. (2004). Reflexiones sobre el estado actual de la ciencia política en Colombia. Papel Político, 16, 9-27.

Losada, R., \& Casas, A. (2008). Enfoques para el análisis político. Bogotá: Universidad Javeriana.

Mejía-Quintana, O. (2006). Estatuto epistemológico de la teoría política: problemática, reconstrucción y competencia. Ciencia Política, 1, 30-57

Mertz, O. (1984). Epistemología y Ciencia Política. Revista de Ciencia Política, 6(2), 47-70.

Monroe, K. (Coord.) (2005). Perestroika! The Raucous Rebellion in Political Science. New Haven: Yale University Press.
Murillo, G., \& Ungar, E. (1999). Evolución y desarrollo de la Ciencia Política colombiana: un proceso en marcha. Revista de Estudios Sociales, 4, 36-53.

Parra, A., Tabares, C., \& Hurtado, D. (2013) ¿De qué Ciencia Política estamos hablando? Las potencialidades de un enfoque integrador. Estudios Politicos, 43, 13-38.

Pierson, P., \& Skocpol, T. (2008). El institucionalismo histórico en la Ciencia Política Contemporánea. Revista Uruguaya de Ciencia Política, 17(1), 7-38.

Resnik, M. (1998). Paradigmas en Ciencia Política. Buenos Aires: Depalma

Roth, A. (2008). Perspectivas teóricas para el análisis de las políticas públicas: ¿de la razón científica al arte retórico?. Estudios Políticos, 33, 67-91.

Sabatier, P. (1999). Theories of the policy process. Boulder: Westview Press.

Sartori, G. (2004). ¿Hacia dónde va la Ciencia Política?. Política y Gobierno, 11(2), 349-354.

Sorauf, F., \& Hyneman, C. (1967). Ciencia Política. Una sencilla visión general. México: Editorial Hispanoamericana.

Strasser, C. (1979). La idea de una ciencia política. Desarrollo Económico. Revista de Ciencias Sociales, 12(46), 405-420.

Zolo, D. (1989). La tragedia de la Ciencia Política. Metapolítica, 49, 51-69. Recuperado de http://rephip.unr.edu.ar/bitstream/ handle/2133/1463/La_tragedia_de_la_ ciencia_politicaTyD14.pdf? sequence $=1$ 
